\title{
Decentralized Multi-Agent Optimization via Dual Decomposition *
}

\author{
Håkan Terelius *, Ufuk Topcu ${ }^{* *}$ and Richard M. Murray ${ }^{* *}$ \\ * ACCESS Linnaeus Centre, Royal Institute of Technology, \\ Stockholm, SE-10044 Sweden (e-mail: hakante@kth.se) \\ ** Control and Dynamical Systems, California Institute of Technology, \\ Pasadena, CA 91125 USA (e-mail: $\{$ utopcu, murray\}@cds.caltech.edu)
}

\begin{abstract}
We study a distributed multi-agent optimization problem of minimizing the sum of convex objective functions. A new decentralized optimization algorithm is introduced, based on dual decomposition, together with the subgradient method for finding the optimal solution. The iterative algorithm is implemented on a multi-hop network and is designed to handle communication delays. The convergence of the algorithm is proved for communication networks with bounded delays. An explicit bound, which depends on the communication delays, on the convergence rate is given. A numerical comparison with a decentralized primal algorithm shows that the dual algorithm converges faster, and with less communication.
\end{abstract}

Keywords: Distributed multi-agent systems, Convex optimization, Dual decomposition

\section{INTRODUCTION}

The ability to compute an optimal distributed decision has gained a lot of attention in recent research, e.g., Tsitsiklis (1984); Lynch (1996); Rabbat and Nowak (2004); Nedić and Ozdaglar (2007); Johansson (2008). Distributed optimization problems appear in a broad range of practical applications, such as when a set of network users are competing for a shared resource, with different individual objectives. These problems are commonly described as a utility maximization problem, where each user has its own utility function. The goal of the network is to assign the resources such that the total utility is maximized. The users are referred to as agents, and the interconnected network of agents is called a multi-agent system. The multi-agent system consists of agents making local decisions, while trying to coordinate the overall objective with the other agents in the system.

Another application that has received a lot of attention is distributed cooperative control of unmanned vehicles, and especially, formation control of such vehicles, e.g., OlfatiSaber and Murray (2004); Fax and Murray (2004); Blondel et al. (2005). For example, using several micro-satellites has the potential for greater functionality and reliability than an individual satellite. Also, formation control of a group of unmanned aircrafts or underwater vehicles can severely increase the efficiency in surveillance and searchand-rescue operations. Common to all these examples is that the optimal control algorithms should be completely distributed, relying on only local information.

Decomposition methods for large-scale optimization has been an important research topic for some time, see Cohen

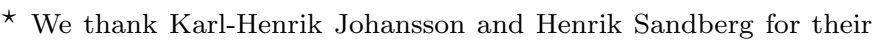
support and helpful discussions. This work was partially supported by the Swedish Research Council, the Knut and Alice Wallenberg Foundation and the Boeing Corporation.
}

(1980), and they can be divided into two approaches: those based on the so called primal and dual decomposition. Nedić and Ozdaglar (2007) have analyzed a decentralized optimization algorithm based on primal decomposition. In this paper, we propose and analyze a decentralized optimization algorithm based on the dual decomposition technique. Both of these algorithms use the subgradient method, a first order optimization method that leads to simple decentralized algorithms. Another advantage of the subgradient method is that it can solve non-smooth optimization problems without prior knowledge of the problem structure, compared to Nesterov (2005).

In Section 2, we describe the optimization problem and the corresponding dual optimization problem. In Section 3 , we develop a novel decentralized algorithm based on the dual optimization problem, and in Section 4, we analyze the convergence rate of the algorithm. In Section 5, we study the communication requirements for the algorithm, and finally, in Section 6, we evaluate the algorithm with numerical simulations. An extended version of this work is available as Terelius (2010).

\section{PROBLEM FORMULATION}

We consider an optimization problem, where a network $\mathcal{G}=(\mathcal{V}, \mathcal{E})($ Fig. 1) of $N$ interconnected agents are minimizing the sum of their individual convex (potentially non-smooth) objective functions $f^{i}: \mathbb{R}^{n} \rightarrow \mathbb{R}$.

$$
f^{*}:=\min _{x \in X} \sum_{i=1}^{N} f^{i}(x),
$$

where $X \subseteq \mathbb{R}^{n}$ is non-empty and convex. Each individual objective function $f^{i}$ is assumed to be known only by agent $i$, thus the agents $\mathcal{V}=\{1, \ldots, N\}$ need to coordinate their decisions through a limited set of communication links 


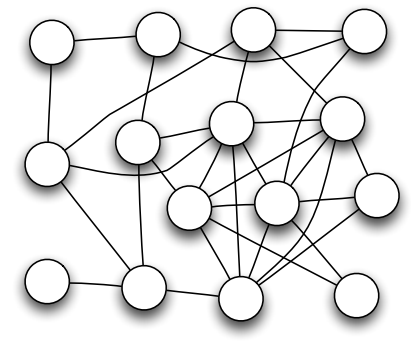

Fig. 1. A multi-agent system $\mathcal{G}=(\mathcal{V}, \mathcal{E})$, where the agents are pictured as vertices $\mathcal{V}$, and the communication links between the agents are pictured as edges $\mathcal{E}$.

$\mathcal{E} \subset \mathcal{V} \times \mathcal{V}$. The goal is to solve the minimization problem in a decentralized fashion, where the agents cooperatively find the optimal solution without a central coordinator. We propose a procedure based on dual decomposition. As a prelude to the proposed procedure, let us discuss the dual decomposition technique.

To this end, let us introduce a local estimate $x^{i} \in X$ of the common decision variable $x \in X$ for each agent $i \in \mathcal{V}$ in the network. The optimization problem in (1) can then be written as

$$
\begin{array}{ll}
\min _{x^{1}, \ldots, x^{N} \in X} & \sum_{i=1}^{N} f^{i}\left(x^{i}\right), \\
\text { subject to } & x^{1}=x^{2}=\cdots=x^{N} .
\end{array}
$$

Notice that the coupling between the objective functions is moved from the decision variable to the consistency constraints. Furthermore, there is redundancy in those constraints, and the question of finding the optimal set of constraints is left as future research. We focus on a subset of the constraints motivated by a partitioning of the decision variable $x \in X$ into $N$ parts, such that each part can be associated with a unique agent.

$$
x=\left[x_{1}^{T}, x_{2}^{T}, \ldots, x_{N}^{T}\right]^{T}
$$

where $x_{i} \in \mathbb{R}^{n_{i}}$ for $i \in \mathcal{V}, n_{i} \geq 0$ and $\sum_{i=1}^{N} n_{i}=n$. This partitioning can be arbitrary, however, in many applications there is a natural partitioning, where $x_{i}$ represents the internal state of agent $i$. Thus, $x_{j}^{i}$ would denote agent $i$ 's estimate of agent $j$ 's internal state. We now introduce the dual variables $\lambda_{i j}, i, j \in \mathcal{V}$, where $\lambda_{i j}$ is associated with the constraint $x_{i}^{i}=x_{i}^{j}$. For notational simplicity, let us also introduce $\lambda$ as

$$
\lambda:=\left[\lambda_{11}^{T}, \ldots, \lambda_{1 N}^{T}, \lambda_{21}^{T}, \ldots, \lambda_{2 N}^{T}, \ldots, \lambda_{N N}^{T}\right]^{T} .
$$

We continue by forming the Lagrangian $L$,

$$
\begin{aligned}
L\left(x^{1}, x^{2}, \ldots, x^{N}, \lambda\right):= & \sum_{i=1}^{N} f^{i}\left(x_{1}^{i}, x_{2}^{i}, \ldots, x_{N}^{i}\right) \\
& +\sum_{i=1}^{N} \sum_{j=1}^{N} \lambda_{i j}^{T}\left(x_{i}^{i}-x_{i}^{j}\right),
\end{aligned}
$$

and the corresponding Lagrange dual function $q$ is the infimum with respect to the primal variables $x^{1}, \ldots, x^{N}$,

$$
q(\lambda):=\inf _{x^{1}, x^{2}, \ldots, x^{N} \in X} L\left(x^{1}, x^{2}, \ldots, x^{N}, \lambda\right) .
$$

Remark 1. The Lagrangian $L$ is independent of the dual variables $\lambda_{i i}$, since $\lambda_{i i}$ accounts for the difference $x_{i}^{i}-$ $x_{i}^{i}=0$. For notational simplicity, we define $\lambda_{i i}=0$.
Let us now rewrite the Lagrange dual function by introducing the subproblems $\phi^{i}(\lambda)$ as

$$
\phi^{i}(\lambda):=\inf _{x^{i} \in X} f^{i}\left(x_{1}^{i}, x_{2}^{i}, \ldots, x_{N}^{i}\right)+\sum_{j=1}^{N} \lambda_{i j}^{T} x_{i}^{i}-\sum_{j=1}^{N} \lambda_{j i}^{T} x_{j}^{i} .
$$

Notice that the subproblem $\phi^{i}$ only depends on the dual variable $\lambda$, and is the infimum over the local variable $x^{i}$. Thus, agent $i$ is able to compute $\phi^{i}(\lambda)$ locally, independent of all other agents. Further, the Lagrange dual function is the sum of all the subproblems,

$$
q(\lambda)=\sum_{i=1}^{N} \phi^{i}(\lambda)
$$

Finally, the Lagrange dual problem is the maximization of the Lagrange dual function,

$$
q^{*}:=\max _{\lambda} q(\lambda)=\max _{\lambda} \sum_{i=1}^{N} \phi^{i}(\lambda) \text {. }
$$

Slater's condition, see Boyd and Vandenberghe (2004), guarantees that $q^{*}=f^{*}$ under certain convexity assumptions. When the optimal dual solution is found, each agent can compute a primal solution by solving (2), moreover, if the problem is strictly convex then all the primal solutions will coincide.

\section{DUAL OPTIMIZATION ALGORITHM}

We solve the Lagrange dual problem (4) with the subgradient method. The subgradient method is a generalization of the gradient descent method to non-differentiable functions, using the iterations

$$
\lambda(t+1)=\lambda(t)+\alpha_{t} g(t)
$$

where $\alpha_{t}$ is the step size used at time $t$, and $g(t)$ is a subgradient to $q$ at $\lambda(t)$, i.e.,

holds for all $\bar{\lambda}$.

$$
q(\bar{\lambda}) \leq q(\lambda(t))+g(t)^{T}(\bar{\lambda}-\lambda(t))
$$

The subgradient update (5) for a single component $\lambda_{i j}$ of the dual variable is $x_{i}^{i}-x_{i}^{j}$, thus the subgradient update can be written as

$$
\lambda_{i j}(t+1)=\lambda_{i j}(t)+\alpha_{t}\left(x_{i}^{i}(t)-x_{i}^{j}(t)\right),
$$

where $x^{i}(t)$ is given by the solution to subproblem $\phi^{i}$, evaluated at $\lambda(t)$.

The subproblems (2) are computationally similar to the primal subproblems of minimizing $f^{i}$, but can be solved independently of each other. The remaining part of the algorithm is to update the dual variables, $\lambda$, in a decentralized manner. We assume that all agents perform their computations and communications synchronously, at the discrete times $t=0,1, \ldots$. Further, we assume that only neighbors can communicate with each other during one time step.

For the subgradient update (7) both agent $i$ 's estimate $x_{i}^{i}$ and agent $j$ 's estimate $x_{i}^{j}$ are necessary to update the dual variable $\lambda_{i j}$. Thus, we let agent $i$ send its estimate to agent $j$, who then is able to update the dual variable. Because the two agents might not be neighbors, we introduce the timedelay $\delta_{i j}$ that measures the multi-hop delay from agent $i$ to 
agent $j$. Further, let $d_{i j}:=\delta_{i j}+\delta_{j i}$ denote the round-trip time between agent $i$ and agent $j$.

The update rule (7) is now modified to account for the communication delays. Let $\lambda_{i j}(t)$ denote the commonly known dual variable that is known to both agent $i$ and agent $j$ at time $t$, which they also use to solve their respective subproblem $\phi^{i}$ and $\phi^{j}$ at time $t$. The solutions to the subproblems, given $\lambda(t)$, is denoted by $x^{i}(t)$ and $x^{j}(t)$. Agent $i$ transmits the part $x_{i}^{i}(t)$ to agent $j$, who receives it at time $t+\delta_{i j}$. Agent $j$ then updates the dual variable $\lambda_{i j}$, which it transmits back to agent $i$. The total time it takes to update the commonly known dual variable is equal to the round trip time $d_{i j}$ between agent $i$ and agent $j$. The update can be formally expressed as

$$
\lambda_{i j}\left(t+d_{i j}+1\right)=\lambda_{i j}\left(t+d_{i j}\right)+\alpha_{t}\left(x_{i}^{i}(t)-x_{i}^{j}(t)\right) .
$$

Remark 2. The commonly known dual variable does not necessarily satisfy the subgradient condition (6), and this is the main problem that is analyzed in the next section.

Remark 3. Different parts of the dual variable $\lambda$ can be updated with different delays, but each part is updated exactly once at each iteration. Also, each agent solves its subproblem exactly once at each iteration.

Before we move into the analysis of the algorithm, we state four assumptions about the problem instance. Assumptions 1-3 are commonly used for the subgradient method and Assumption 4 is due to the time-delays we introduced. Assumption 1. (Existence of Maximizer). There exists at least one finite maximizer of $q$, denoted by $\lambda^{*}$. Let $\Lambda_{o p t}$ denote the set of maximizers to $q$.

Assumption 2. (Bounded Subgradients). For every subgradient $g(t)$ to $q$ at $\lambda(t), g(t)$ is uniformly bounded by G,

$$
\|g(t)\|_{2} \leq G \quad \forall t
$$

Remark 4. Assumption 2 especially holds if the set $X$ is compact.

Assumption 3. (Bounded Initial Distance). The distance from the initial point, $\lambda(0)$, to the optimal set is bounded by $R$,

$$
d\left(\lambda(0), \Lambda_{\text {opt }}\right) \leq R
$$

In order for the algorithm to work, it is necessary to assume that the network is strongly connected. We impose this by assuming that the time-delays are bounded, which also implicitly implies that no transmissions are lost in the network.

Assumption 4. (Bounded Time-Delays). There exists an upper bound $D$ on the round-trip time,

$$
d_{i j}=\delta_{i j}+\delta_{j i} \leq D \quad \forall i, j \in \mathcal{V} .
$$

\section{CONVERGENCE ANALYSIS}

The analysis focuses on the evolution of the dual variables $\lambda(t)$, recall that $g(t)$ is a subgradient to the Lagrange dual function, $q$, evaluated at $\lambda(t)$. Let us define the shifted vector of dual variables $\bar{\lambda}(t)$ by

$$
\bar{\lambda}_{i j}(t):=\lambda_{i j}\left(t+d_{i j}\right),
$$

thus, the entire vector $\bar{\lambda}(t)$ is

$$
\bar{\lambda}(t)=\left[\begin{array}{c}
\lambda_{11}\left(t+d_{11}\right) \\
\vdots \\
\lambda_{i j}\left(t+d_{i j}\right) \\
\vdots \\
\lambda_{N N}\left(t+d_{N N}\right)
\end{array}\right] .
$$

With this notation the update rule (8) can be written as

$$
\bar{\lambda}(t+1)=\bar{\lambda}(t)+\alpha_{t} g(t) \text {. }
$$

Remark 5. Notice the similarity between this expression and the ordinary subgradient iteration (5), however, remember that $g(t)$ is a subgradient at $\lambda(t)$ and not at $\bar{\lambda}(t)$.

The entire dual vector $\lambda(t)$ can be expressed in terms of $\bar{\lambda}(t)$ with the following vector projection $P_{d}$. Let $P_{d}(x)$ be defined by

$$
\left[P_{d}(x)\right]_{i j}:= \begin{cases}x_{i j} & \text { if } d_{i j}=d ; \\ 0 & \text { otherwise. }\end{cases}
$$

In other words, $P_{d}(x)$ selects those components of $x$ whose round-trip time is equal to $d$. Under Assumption $4 P_{d}$ has two important properties; first, for any vector $x$

$$
\sum_{d=0}^{D} P_{d}(x)=x
$$

Second, we can express $\lambda(t)$ from $\bar{\lambda}(t)$ as

$$
\sum_{d=0}^{D} P_{d}(\bar{\lambda}(t-d))=\lambda(t)
$$

Lemma 1. Under Assumption 3,

$$
d\left(\bar{\lambda}(0), \Lambda_{\text {opt }}\right) \leq R
$$

Proof. By the definition of $\bar{\lambda}$, we have

$$
\bar{\lambda}_{i j}(0)=\lambda_{i j}\left(d_{i j}\right) \text {. }
$$

Notice that it takes $d_{i j}$ time steps until agent $i$ receives its first update on the dual variable $\lambda_{i j}$, hence $\lambda_{i j}(0)=$ $\cdots=\lambda_{i j}\left(d_{i j}\right)$ and $\bar{\lambda}(0)=\lambda(0)$. The lemma now follows from Assumption 3.

Lemma 2. Under Assumption 2,

$$
\|\bar{\lambda}(t+1)-\bar{\lambda}(t)\|_{2} \leq \alpha_{t} G \quad \forall t .
$$

Proof. From the update rule (9), we have

$$
\bar{\lambda}(t+1)-\bar{\lambda}(t)=\alpha_{t} g(t)
$$

Thus, by Assumption 2,

$$
\|\bar{\lambda}(t+1)-\bar{\lambda}(t)\|_{2}=\alpha_{t}\|g(t)\|_{2} \leq \alpha_{t} G
$$

In the next lemma we give an upper bound on the difference between $\bar{\lambda}(t)$ and $\lambda(t)$.

Lemma 3. Under Assumptions 2 and 4,

$$
\|\bar{\lambda}(t)-\lambda(t)\|_{2} \leq G \sum_{d=t-D}^{t-1} \alpha_{d} \quad \forall t .
$$

Proof. Recall that $\bar{\lambda}(t)-\lambda(t)$ can be written as

$$
\begin{aligned}
\bar{\lambda}(t)-\lambda(t) & =\bar{\lambda}(t)-\sum_{d=0}^{D} P_{d}(\bar{\lambda}(t-d)) \\
& =\sum_{d=1}^{D} P_{d}(\bar{\lambda}(t)-\bar{\lambda}(t-d)) .
\end{aligned}
$$


By replacing the term $\bar{\lambda}(t)-\bar{\lambda}(t-d)$ with a telescoping sum, we have

$$
\begin{aligned}
\bar{\lambda}(t)-\lambda(t) & =\sum_{d=1}^{D} P_{d}\left(\sum_{i=0}^{d-1}(\bar{\lambda}(t-i)-\bar{\lambda}(t-i-1))\right) \\
& =\sum_{d=1}^{D} \sum_{i=0}^{d-1} P_{d}(\bar{\lambda}(t-i)-\bar{\lambda}(t-i-1)),
\end{aligned}
$$

since $P_{d}$ is a linear function. Now, by changing the order of summation in this double sum,

$$
\bar{\lambda}(t)-\lambda(t)=\sum_{i=0}^{D-1} \sum_{d=i+1}^{D} P_{d}(\bar{\lambda}(t-i)-\bar{\lambda}(t-i-1)) .
$$

By the triangle inequality,

$\|\bar{\lambda}(t)-\lambda(t)\|_{2} \leq \sum_{i=0}^{D-1}\left\|\sum_{d=i+1}^{D} P_{d}(\bar{\lambda}(t-i)-\bar{\lambda}(t-i-1))\right\|_{2}$

Further, since the projections $P_{d}$ are disjoint for different $d$,

$$
\begin{aligned}
& \left\|\sum_{d=i+1}^{D} P_{d}(\bar{\lambda}(t-i)-\bar{\lambda}(t-i-1))\right\|_{2} \\
& \leq\left\|\sum_{d=0}^{D} P_{d}(\bar{\lambda}(t-i)-\bar{\lambda}(t-i-1))\right\|_{2} \\
& =\|\bar{\lambda}(t-i)-\bar{\lambda}(t-i-1)\|_{2} .
\end{aligned}
$$

Using Lemma 2 yields

$$
\|\bar{\lambda}(t-i)-\bar{\lambda}(t-i-1)\|_{2} \leq G \alpha_{t-i-1} .
$$

Assembling everything together gives us

$$
\|\bar{\lambda}(t)-\lambda(t)\|_{2} \leq \sum_{i=0}^{D-1} G \alpha_{t-i-1}=G \sum_{d=t-D}^{t-1} \alpha_{d} .
$$

Remark 6. For $t<D, \lambda(t)=\sum_{d=0}^{t} P_{d}(\bar{\lambda}(t-d))$ and the bound in Lemma 3 becomes

$$
\|\bar{\lambda}(t)-\lambda(t)\|_{2} \leq G \sum_{d=0}^{t-1} \alpha_{d}
$$

Instead of treating this case separately, we define $\alpha_{t}=0$ for $t<0$.

Lemma 4. Under Assumptions 2 and 4,

$$
g(t)^{T}\left(\bar{\lambda}(t)-\lambda^{*}\right) \leq G^{2} \sum_{d=t-D}^{t-1} \alpha_{d}+q(\lambda(t))-q^{*} \quad \forall t .
$$

Proof. Adding $g(t)^{T}(\lambda(t)-\lambda(t))=0$ to the left-hand side of equation (12) yields

$g(t)^{T}\left(\bar{\lambda}(t)-\lambda^{*}\right)=g(t)^{T}(\bar{\lambda}(t)-\lambda(t))+g(t)^{T}\left(\lambda(t)-\lambda^{*}\right)$

By Cauchy-Schwarz inequality,

$$
g(t)^{T}(\bar{\lambda}(t)-\lambda(t)) \leq\|g(t)\|_{2} \cdot\|\bar{\lambda}(t)-\lambda(t)\|_{2} .
$$

Since $g(t)$ is a subgradient at $\lambda(t)$, the subgradient definition (6) implies that

Thus,

$$
g(t)^{T}\left(\lambda(t)-\lambda^{*}\right) \leq q(\lambda(t))-q^{*} .
$$

$g(t)^{T}\left(\bar{\lambda}(t)-\lambda^{*}\right) \leq\|g(t)\|_{2} \cdot\|\bar{\lambda}(t)-\lambda(t)\|_{2}+q(\lambda(t))-q^{*}$.

Finally, using Lemma 3 and Assumption 2, we get

$$
g(t)^{T}\left(\bar{\lambda}(t)-\lambda^{*}\right) \leq G^{2} \sum_{d=t-D}^{t-1} \alpha_{d}+q(\lambda(t))-q^{*}
$$

The function value $q(\lambda(t))$ does not have to be monotonically increasing. Therefore, the algorithm is evaluated as the maximum value over a period of $T$ iterations. Let us now state and prove the main convergence theorem for the dual optimization algorithm.

Theorem 5. Let Assumptions 1,2, 3 and 4 hold. Then, the maximum value satisfies the following bound,

$\max _{t=0, \ldots, T} q(\lambda(t)) \geq q^{*}-\frac{R^{2}+G^{2} \sum_{t=0}^{T}\left(\alpha_{t}^{2}+2 \alpha_{t} \sum_{d=t-D}^{t-1} \alpha_{d}\right)}{2 \sum_{t=0}^{T} \alpha_{t}}$.

Proof. Let $\lambda^{*}$ be any optimal point to $q$, and let $\bar{\lambda}(t)$ be given by the iterations (9). Consider the following relation

$$
\begin{aligned}
0 & \leq\left\|\bar{\lambda}(T+1)-\lambda^{*}\right\|_{2}^{2}=\left\|\bar{\lambda}(T)+\alpha_{T} g(T)-\lambda^{*}\right\|_{2}^{2} \\
& =\left\|\bar{\lambda}(T)-\lambda^{*}\right\|_{2}^{2}+2 \alpha_{T} g(T)^{T}\left(\bar{\lambda}(T)-\lambda^{*}\right)+\alpha_{T}^{2}\|g(T)\|_{2}^{2} .
\end{aligned}
$$

This is a recursive equation in $\left\|\bar{\lambda}(t)-\lambda^{*}\right\|_{2}^{2}$, and can be expanded until $t=0$, yielding

$0 \leq\left\|\bar{\lambda}(0)-\lambda^{*}\right\|_{2}^{2}+\sum_{t=0}^{T} 2 \alpha_{t} g(t)^{T}\left(\bar{\lambda}(t)-\lambda^{*}\right)+\sum_{t=0}^{T} \alpha_{t}^{2}\|g(t)\|_{2}^{2}$.

Using equation (12) from Lemma 4 gives us

$$
\begin{gathered}
0 \leq\left\|\bar{\lambda}(0)-\lambda^{*}\right\|_{2}^{2}+\sum_{t=0}^{T} 2 \alpha_{t}\left(G^{2} \sum_{d=t-D}^{t-1} \alpha_{d}+q(\lambda(t))-q^{*}\right) \\
+\sum_{t=0}^{T} \alpha_{t}^{2}\|g(t)\|_{2}^{2} \\
\leq\left\|\bar{\lambda}(0)-\lambda^{*}\right\|_{2}^{2}+\sum_{t=0}^{T} 2 \alpha_{t} G^{2} \sum_{d=t-D}^{t-1} \alpha_{d} \\
+\left(\max _{t=0, \ldots, T} q(\lambda(t))-q^{*}\right) \sum_{t=0}^{T} 2 \alpha_{t}+\sum_{t=0}^{T} \alpha_{t}^{2}\|g(t)\|_{2}^{2} .
\end{gathered}
$$

Thus,

$$
\begin{array}{rl}
\max _{t=0, \ldots, T} & q(\lambda(t)) \geq q^{*}-\frac{\left\|\bar{\lambda}(0)-\lambda^{*}\right\|_{2}^{2}}{\sum_{t=0}^{T} 2 \alpha_{t}} \\
- & \frac{\sum_{t=0}^{T} 2 \alpha_{t} G^{2} \sum_{d=t-D}^{t-1} \alpha_{d}+\sum_{t=0}^{T} \alpha_{t}^{2}\|g(t)\|_{2}^{2}}{\sum_{t=0}^{T} 2 \alpha_{t}} .
\end{array}
$$

Notice that $\lambda^{*}$ is an arbitrary optimal point, thus $\left\|\bar{\lambda}(0)-\lambda^{*}\right\|_{2}$ can be replaced with $d\left(\bar{\lambda}(0), \Lambda_{\text {opt }}\right)$. Finally, using Lemma 1 and Assumption 2 gives us

$$
\begin{aligned}
& \max _{t=0, \ldots, T} q(\lambda(t)) \\
& \geq q^{*}-\frac{R^{2}+\sum_{t=0}^{T} 2 \alpha_{t} G^{2} \sum_{d=t-D}^{t-1} \alpha_{d}+\sum_{t=0}^{T} \alpha_{t}^{2} G^{2}}{\sum_{t=0}^{T} 2 \alpha_{t}} \\
& =q^{*}-\frac{R^{2}+G^{2} \sum_{t=0}^{T}\left(\alpha_{t}^{2}+2 \alpha_{t} \sum_{d=t-D}^{t-1} \alpha_{d}\right)}{2 \sum_{t=0}^{T} \alpha_{t}} .
\end{aligned}
$$

Corollary 6. Let Assumptions 1 - 4 hold, then, for a constant step size $\alpha_{t}=\alpha$,

$$
\max _{t=0, \ldots, T-1} q(\lambda(t)) \geq q^{*}-\frac{R^{2}+G^{2} \alpha^{2} T(1+2 D)}{2 \alpha T} .
$$

By letting $D=0$ in Corollary 6, we recognize the convergence result for the ordinary subgradient method, 
without any delays. The conclusion is that introducing communication delays do not affect the convergence speed, but instead causes the algorithm to converge to a larger neighborhood around the optimal solution. The intuition behind the result is that the ordinary subgradient method can overshoot the optimal point with half the step length, while using delayed information can cause the algorithm to continue past the optimal point for an additional $D$ steps. Corollary 7. Consider also a square summable, but not summable step size $\alpha_{t} \geq \alpha_{t+1} \geq 0 \quad t=0,1, \ldots$, such that $\sum_{t=0}^{\infty} \alpha_{t}^{2}<\infty$ and $\sum_{t=0}^{\infty} \alpha_{t}=\infty$. Let Assumptions $1-4$ hold, then

$\max _{t=0, \ldots, T} q(\lambda(t)) \geq q^{*}-\frac{R^{2}+G^{2}(1+2 D) \sum_{i=0}^{T} \alpha_{i-D}^{2}}{2 \sum_{i=0}^{T} \alpha_{i}} \rightarrow q^{*}$ as $T \rightarrow \infty$. Thus, this choice of step size guarantees asymptotic convergence to the optimal solution.

\section{COMMUNICATION COST}

In distributed systems, such as wireless sensor networks, the limiting resource is often the communication, Akyildiz et al. (2002). It is therefore important to analyze the convergence speed in relation to the communication. For simplicity, we define the communication cost of a transmission as the number of real valued scalars transferred. There are two contributions to the communication cost: distributing the primal variables $x_{i}^{i}(t)$, and accumulating the dual variables $\lambda_{j i}$. Let us analyze these contributions separately, per iteration of the algorithm.

Recall that only the part $x_{i}^{i}(t)$ of agent $i$ 's estimate $x^{i}(t)$ has to be distributed to all other agents in the network, in order to update the dual variables. A reasonable assumption is that an agent only receives the estimate $x_{i}^{i}(t)$ once. Since there are $N$ agents in the network, each part is transferred over exactly $N-1$ edges. Summing over all parts yields the communication cost

$$
\sum_{i=1}^{N}(N-1) \operatorname{dim}\left(x_{i}^{i}\right)=(N-1) \operatorname{dim}(x)=(N-1) n .
$$

We similarly have the communication cost for the dual variables

$$
\sum_{i=1}^{N} \sum_{j=1}^{N}(N-1) \operatorname{dim}\left(\lambda_{i j}\right)=(N-1) \operatorname{dim}(\lambda) .
$$

Compared to the primal variables, there are many more dual variables. To improve upon this, notice that the subproblem $\phi^{i}(\lambda)$ only depends on the dual variables $\lambda_{j i}$, $j \in \mathcal{V}$, and the sum $\sum_{j=1}^{N} \lambda_{i j}$, not the individual values $\lambda_{i j}$, $j \in \mathcal{V}$. Further, since the dual variables $\lambda_{j i}$ are calculated at agent $i$, it only needs to receive the sum of the dual variables $\sum_{j=1}^{N} \lambda_{i j}$.

Thus, an agent $k$ sums all the received dual variables $\lambda_{i}$. directed towards agent $i$, add its own dual variable $\lambda_{i k}$ to this sum, and then transfer the entire sum instead of the individual values. All dual variables $\lambda_{i}$. directed towards agent $i$ are then together only transferred over $N-1$ edges, and the total cost of transporting all dual variables is

$$
\sum_{i=1}^{N}(N-1) \operatorname{dim}\left(\lambda_{i} .\right)=\sum_{i=1}^{N}(N-1) \operatorname{dim}\left(x_{i}\right)=(N-1) n
$$

Adding the communication cost for the primal and dual variables leads to the following proposition.

Proposition 8. The total communication cost for the dual optimization algorithm is

$$
2(N-1) n \text {. }
$$

Remark \%. By aggregating the dual variable updates, and neglecting the cost for routing overhead, the communication cost becomes independent of the network topology.

Further inspection of the problem structure can reduce the communication cost. Recall that each local objective function $f^{i}$ is assumed to depend on all of the decision variables $x_{1}, x_{2}, \ldots, x_{N}$. In practice, many interesting distributed optimization problems have a sparse structure in the dependencies. When $f^{i}$ does not depend on $x_{j}$ then the local estimate $x_{j}^{i}$ is not needed, and neither is the dual variable $\lambda_{j i}$ accounting for the difference between $x_{j}^{j}$ and $x_{j}^{i}$. The implication is that the primal variable $x_{j}^{j}$ does not have to be sent to agent $i$, and the dual variable $\lambda_{j i}$ does not have to be sent back to agent $j$, resulting in a reduced communication cost.

\section{NUMERICAL RESULTS}

\subsection{A Primal Distributed Optimization Algorithm}

We compare the dual optimization algorithm against a decentralized optimization algorithm, analyzed by Nedić and Ozdaglar (2007). We refer to this algorithm as the primal algorithm. The primal algorithm is also an iterative algorithm, where each agent has a local estimate $x^{i}(t)$ of the decision variable, but the update consists of an average consensus update together with a subgradient step for the local objective function, i.e.,

$$
x^{i}(t+1)=\sum_{j=1}^{N} W_{j, i}(t) x^{j}(t)-\alpha_{t}^{i} g^{i}(t),
$$

where $W$ is an average consensus matrix, and $g^{i}(t)$ is a subgradient to $f^{i}$ at $x^{i}(t)$.

\subsection{Evaluating the Convergence Rate}

In order to compare the convergence rate, we need a measure of convergence. For both algorithms, each agent has an estimate of the entire primal decision variable $x^{i}(t)$, but we are seeking a solution where all these estimates are equal. Therefore, we evaluate the algorithms on the average state estimate,

$$
x_{\mathrm{avg}}(t):=\frac{1}{N} \sum_{i=1}^{N} x^{i}(t) .
$$

The function value is then evaluated as

$$
f(t):=\sum_{i=1}^{N} f^{i}\left(x_{\mathrm{avg}}(t)\right) .
$$

Finally, since the function value does not need to be monotonically decreasing, we evaluate each algorithm as the minimum value attained until time $T$,

$$
f_{\text {best }}(T):=\min _{t=0, \ldots, T} f(t) \text {. }
$$


For the dual optimization algorithm, we can also evaluate the Lagrange dual function on the commonly known dual variable $\lambda(t)$,

$$
q(t):=\sum_{i=1}^{N} \phi^{i}(\lambda(t)) .
$$

Also, since the dual function does not need to be monotonically increasing, we evaluate the dual value as the maximum attained until time $T$,

$$
q_{\text {best }}(T):=\max _{t=0, \ldots, T} q(t) .
$$

Both algorithms' convergence rate depends on their respective step size rule. We limit both algorithms to use a constant step size $\alpha_{t}=\alpha$, and then selects the optimal constant step size for each algorithm. Thus, for a given time interval $0, \ldots, T$, we use a step size $\alpha$ such that $f_{\text {best }}(T)$ is minimal.

\subsection{Connected Graph}

We start with a randomly chosen, strongly connected, network consisting of 15 agents (Fig. 1). The objective functions $f^{i}$ are chosen as random convex quadratic functions, where the decision variable is constrained to a compact set $X$, and the initial values are set to zero, $x(0)=0, \lambda(0)=0$. The maximal delay between any two agents is 3 , and hence, the upper bound on the round-trip time is $D=6$. The step sizes are chosen such that the best function value $f_{\text {best }}(T)$ at time step $T=1000$ is minimal. This results in the primal algorithm using the step size $\alpha=0.00069$, and the dual algorithm using the step size $\alpha=0.0568$.

The convergence rate is evaluated on the time interval $t=0, \ldots, 2000$ (Fig. 2). Notice that the primal algorithm reaches a neighborhood around the optimal value at time step 1000, and does not significantly improve during the next 1000 steps. It is a tradeoff in the step size; a smaller step size would yield a slower convergence, but to a value closer to the optimal, thus, giving a worse solution at time step 1000 but a better solution at time step 2000. The dual algorithm, on the other hand, is close to the optimal value already after 1000 steps, and has essentially reached the optimal value after 2000 steps.

Finally, let us compare the communication cost for each algorithm. Notice that each agent in the primal algorithm transmits its local estimate to all available neighbors, hence the communication cost for the primal algorithm is $|\mathcal{E}| \operatorname{dim}(x)$, where the edges are counted with direction. The network in Fig. 1 has 62 edges, counted once in each direction, and hence, the communication cost is $62 n$ for the primal, and $28 n$ for the dual algorithm.

Thus, for this problem instance, the dual algorithm converges faster, to a smaller neighborhood around the optimal value, and with less communication than the primal algorithm.

\subsection{Line Graph}

Next, we consider a network with a line topology (Fig. 3). A line graph with $N$ agents is a connected graph with diameter $N-1$, and thus, has the highest roundtrip time among all connected graphs of $N$ agents. The

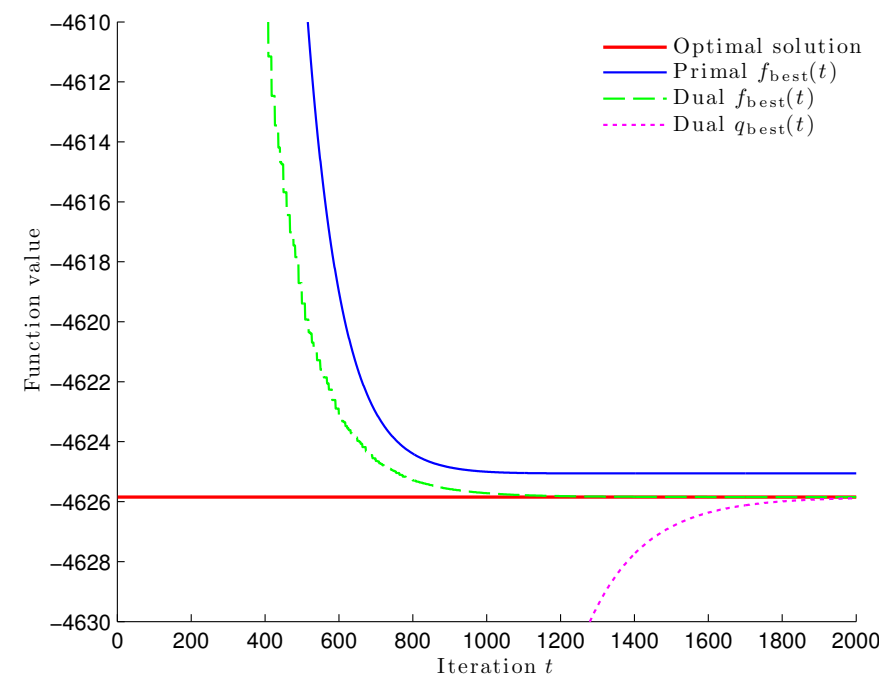

Fig. 2. Convergence simulation for agents communicating through a connected network (Fig. 1). The primal algorithm reaches a neighborhood around the optimal value, while the dual algorithm converges to the optimal value.

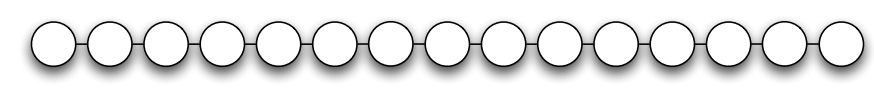

Fig. 3. The line topology has the maximal round-trip time among all networks with $N$ agents.

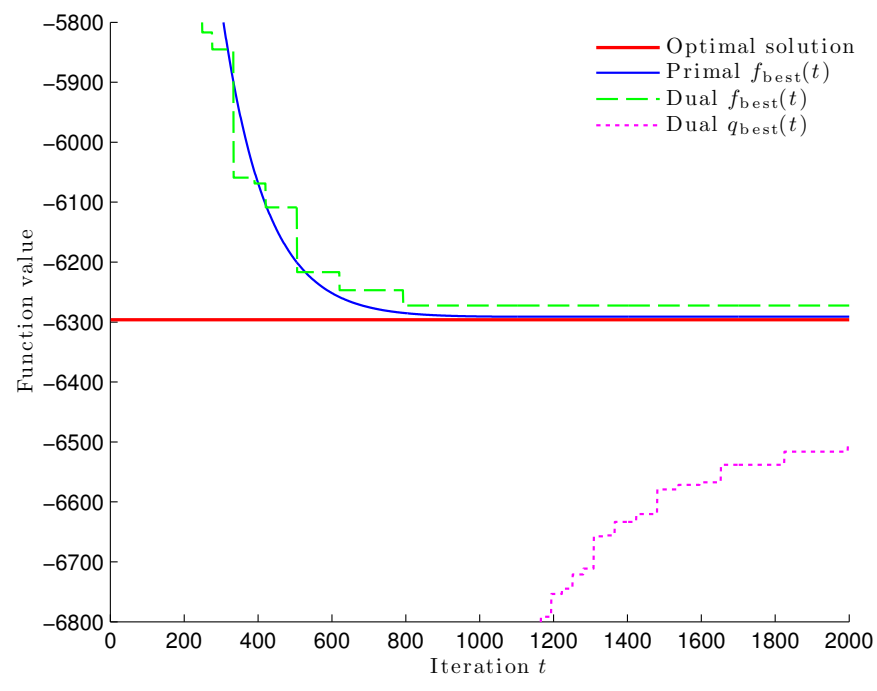

Fig. 4. Convergence simulation for agents communicating through a line topology (Fig. 3). The primal algorithm attains a lower value than the dual algorithm. The staircase characteristics of the dual algorithm is typical for oscillations in the function value $f(t)$, caused by the delays.

round-trip time is bounded by $D=26$. The optimization problem is otherwise chosen as in the previous section, with the optimal step sizes chosen at time $T=1000$. The convergence rate is once again evaluated on the time interval $t=0, \ldots, 2000$ (Fig. 4).

The results here are almost the opposite compared to the previous example. The primal algorithm converges to a 


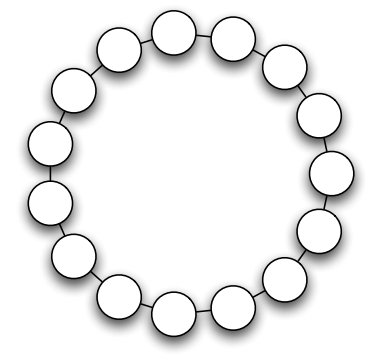

Fig. 5. The circular topology has one additional edge compared to the line topology, cutting the round-trip time in half.

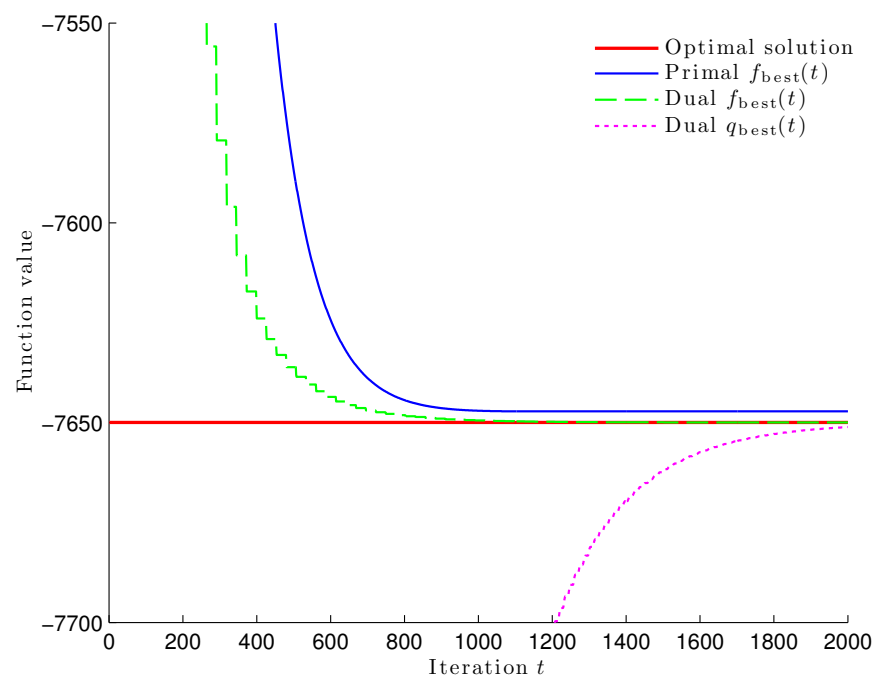

Fig. 6. Convergence simulation for agents communicating through a circular topology (Fig. 5). The primal algorithm has reached a neighborhood around the optimal value, while the dual algorithm converges to the optimal value.

lower value than the dual algorithm. The dual algorithm, on the other hand, reaches a neighborhood around the optimal value before step 1000, and does not improve after that. Notice the characteristic staircase appearance in the graph, it is caused by oscillations in the underlying function values $f(t)$, which are due to the delays in the network. Further, the number of directed edges is 28 , thus the communication cost for both the primal and dual algorithm are equal to $28 n$.

Thus, for this problem, the primal algorithm converges to a smaller neighborhood around the optimal value, and with the same communication cost as the dual algorithm.

\subsection{Circular Graph}

As a last example, we consider a network with circular topology (Fig. 5), which can be obtained from the line graph by adding only a single edge. This edge, however, causes the diameter of the graph to shrink from 14 to 7 , and the upper bound on the round-trip time is $D=12$ instead of 26 . The optimization problem is again chosen as in the previous sections, and the convergence rate is evaluated on the interval $t=0, \ldots, 2000$ (Fig. 6).
The results are similar to those of the first example, where the primal algorithm reaches a neighborhood around the optimal value before step 1000, while the dual algorithm converges to the optimal value. Also, the communication cost for the primal algorithm is $30 n$ compared to the $28 n$ for the dual algorithm.

\section{CONCLUSIONS}

Distributed optimization problems appear in a broad range of practical applications. In this paper, we have developed a novel decentralized optimization algorithm, based on the dual decomposition technique, capable of handling communication delays. Our analysis of the algorithm shows that it converges to the optimal solution when the communication delay is bounded. The numerical simulations show some promising results for our algorithm, both in terms of convergence speed and in decreasing the communication cost, especially for networks with a small diameter.

\section{REFERENCES}

Akyildiz, I.F., Su, W., Sankarasubramaniam, Y., and Cayirci, E. (2002). Wireless sensor networks: a survey. Computer Networks, 38(4), 393-422.

Blondel, V.D., Hendrickx, J.M., Olshevsky, A., and Tsitsiklis, J.N. (2005). Convergence in multiagent coordination, consensus, and flocking. In Proceedings of Joint 44th IEEE Conference on Decision and Control and European Control Conference, 2996-3000.

Boyd, S. and Vandenberghe, L. (2004). Convex Optimization. Cambridge University Press.

Cohen, G. (1980). Auxiliary problem principle and decomposition of optimization problems. Journal of Optimization Theory and Applications, 32, 277-305.

Fax, J.A. and Murray, R.M. (2004). Information flow and cooperative control of vehicle formations. IEEE Transactions on Automatic Control, 49(9), 1465-1476.

Johansson, B. (2008). On Distributed Optimization in Networked Systems. Ph.D. thesis, Royal Institute of Technology (KTH). TRITA-EE 2008:065.

Lynch, N.A. (1996). Distributed algorithms. Morgan Kaufmann.

Nedić, A. and Ozdaglar, A. (2007). On the rate of convergence of distributed subgradient methods for multiagent optimization. In Proceedings of 46 th IEEE Conference on Decision and Control, 4711-4716.

Nesterov, Y. (2005). Smooth minimization of non-smooth functions. Mathematical Programming, 103, 127-152.

Olfati-Saber, R. and Murray, R.M. (2004). Consensus problems in networks of agents with switching topology and time-delays. IEEE Transactions on Automatic Control, 49(9), 1520-1533.

Rabbat, M. and Nowak, R. (2004). Distributed optimization in sensor networks. In Proceedings of the $3 \mathrm{rd}$ international symposium on Information processing in sensor networks, IPSN '04, 20-27. ACM.

Terelius, H. (2010). Distributed Multi-Agent Optimization via Dual Decomposition. Master's thesis, Royal Institute of Technology, Stockholm. XR-EE-RT 2010:013.

Tsitsiklis, J.N. (1984). Problems in Decentralized Decision Making and Computation. Ph.D. thesis, Department of EECS, MIT. 